AperTO - Archivio Istituzionale Open Access dell'Università di Torino

\title{
Doxorubicin-Resistant Osteosarcoma: novel therapeutic approaches in sight?
}

\section{This is a pre print version of the following article:}

Original Citation:

\section{Availability:}

This version is available http://hdl.handle.net/2318/1627490

since 2017-05-13T21:45:48Z

Published version:

DOI:10.2217/fon-2016-0519

Terms of use:

Open Access

Anyone can freely access the full text of works made available as "Open Access". Works made available under a Creative Commons license can be used according to the terms and conditions of said license. Use of all other works requires consent of the right holder (author or publisher) if not exempted from copyright protection by the applicable law. 


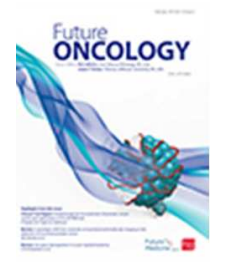

Doxorubicin-Resistant Osteosarcoma: novel therapeutic approaches in sight?

\begin{tabular}{|r|l|}
\hline Journal: & Future Oncology \\
\hline Manuscript ID & Draft \\
\hline Manuscript Type: & Commentary \\
\hline Keywords: & Osteosarcoma, Doxorubicin, Drug resistance \\
\hline \multicolumn{2}{l}{} \\
\end{tabular}

\section{SCHOLARONE ${ }^{\text {Th }}$ \\ Manuscripts}




\section{Doxorubicin-resistant osteosarcoma: novel therapeutic approaches in sight?}

High-grade osteosarcoma (HGOS) is the most common malignant tumour of bone. One of the most active drugs for HGOS treatment is doxorubicin, which is invariably included in HGOS chemotherapy protocols together with methotrexate and cisplatin, with the possible addition of ifosfamide. Several studies have shown that HGOS patients may be inherently resistant to doxorubicin or may become unresponsive to this drug during chemotherapeutic treatment $[1,2]$. The most relevant mechanism of doxorubicin resistance in HGOS has been demonstrated to be the ATP-binding cassette $(\mathrm{ABC})$ transporters-mediated drug efflux, with ABCB1 (P-glycoprotein, MDR1) playing a predominat role [2, 3]. Furthermore, ABCB1 overexpression has been reported independently by several authors to be associated with an adverse clinical outcome $[1,2]$. Based on this evidence, in 2011, the evaluation of ABCB1 protein expression level at diagnosis has been introduced to tailor the treatment of HGOS patients according to their ABCB1 expression status (ClinicalTrials.gov Identifier: NCT01459484). However, in this trial, which is presently ongoing and recruiting patients, $\mathrm{ABCB} 1$ expression level is used to stratify HGOS patients but not as therapeutic target.

A possible strategy to overcome the clinical resistance against doxorubicin may be based on the use of $\mathrm{ABC}$ transporter inhibitors, with the aim to revert doxorubicin-resistant tumour cells toward a drug-sensitive phenotype. Although several $\mathrm{ABC}$ transporter inhibitors have entered phase I-II-III trials for different human tumours, their clinical use has invariably been limited by the severe toxicity exerted at the concentrations required to efficiently inhibit $\mathrm{ABC}$ transporter activity [4]. In the past ten years, in order to overcome this limitation, a new generation of $A B C$ transporter inhibitors has been developed and characterised [4], showing promising preclinical results also in HGOS cells [3].

In the next years, this new generation of $\mathrm{ABCB} 1$ inhibitors might therefore provide novel agents that may be considered for doxorubicin-unresponsive HGOS patients.

An alternative strategy that has been suggested to overcome the ABCB1-mediated drug resistance may be based on the use of curcumin, a phenolic compound used in the traditional indian medicine, which was shown to down-modulate the function of $\mathrm{ABCB} 1$ and to partially revert doxorubicin resistance in human HGOS cell lines [3]. Some years ago, curcumin was included in a phase I-II clinical trial for relapsed or metastatic HGOS patients (ClinicalTrials.gov Identifier: NCT00689195). The recruitment status of this trial is unfortunately unknown and no 
results have been posted yet. It is therefore impossible to understand whether this approach might be of help to overcome the ABCB1-mediated drug resistance in HGOS patients.

Another additional possibility to interfere with $\mathrm{ABCB} 1$ activity in human tumour cells has been highlighted by recent studies on protein kinase inhibitor drugs. Some of them proved to downregulate $\mathrm{ABCB} 1$ activity and, therefore, act as chemoresistance revertants [5]. We have recently provided evidence that this situation also occurs in HGOS cells, and that the combined treatment of specific kinase inhibitor drugs with doxorubicin can overcome the ABCB1-mediated drug resistance in human HGOS cell lines [6, 7]. This may represent a real perspective of intervention to improve the treatment response and outcome of doxorubicin-resistant HGOS patients.

Doxorubicin-unresponsive HGOS patients may be also treated with alternative, conventional chemotherapeutic drugs. There are, in fact, clinical studies demonstrating that HGOS patients may be efficiently treated with etoposide, methotrexate, and ifosfamide $[8,9]$.

In a French randomized trial that enrolled 234 children/adolescents with localized HGOS, the efficacy of preoperative chemotherapy with high-dose methotrexate plus doxorubicin was compared to that of high-dose methotrexate plus etoposide and ifosfamide [8]. A good histological response (tumour necrosis greater than 95\%) was achieved in $56 \%$ of the etoposide-ifosfamide arm patients versus $39 \%$ of the doxorubicin arm $(P=0.009)$. Although no significant difference was found regarding five-year event-free and overall survival between the two protocol arms, this study showed that treatment with methotrexate, etoposide and ifosfamide is effective and can lead to survival rates similar to those achievable with standard regimens based on methotrexate and doxorubicin.

The same group also performed the multicenter OS2006 phase III study, which enrolled 318 HGOS patients and was primarily aimed to assess the efficacy of zoledronic acid [9]. Preoperative chemotherapy addressed to pediatric patients consisted in methotrexate, ifosfamide and etoposide, whereas adult patients received ifosfamide, doxorubicin and cisplatin. Patients with a good histological response received a postoperative treatment with the same drugs as in the preoperative phase. Poor responder pediatric patients were treated with a regimen in which ifosfamide and etoposide were replaced by cisplatin and doxorubicin, whereas poor responder adult patients received etoposide plus ifosfamide. Patients were randomly assigned to receive the aforementioned chemotherapy with or without ten zoledronate intravenous infusions.

Unfortunately, the addition of zoledronic acid did not improve treatment efficacy and prognosis, but the observation that the rate of good histological response was higher in patients treated with 
methotrexate, ifosfamide and etoposide compared to those who received ifosfamide, doxorubicin and cisplatin, confirmed the previous findings reported by the same group [8].

Approaches based on the use of doxorubicin derivatives aiming to improve the local efficacy of the native drug, have also been considered to treat unresponsive, relapsed HGOS patients.

Doxil (liposomal doxorubicin hydrochloride) is a liposomal encapsulated doxorubicin with a longer half-life in blood and an improved drug incorporation into tumour cells.

Doxil was first used in a group of 47 sarcoma patients (including six HGOS) who were unresponsive to doxorubicin and standard chemotherapy [10]. Clinical benefit was observed in three of the six HGOS patients. All of them had previously received doxorubicin and ifosfamide, indicating that doxil may be considered for treating patients refractory to conventional doxorubicin [10].

After this study, doxil has been considered for other clinical trials for patients with HGOS (ClinicalTrials.gov Identifiers: NCT00949325; NCT00019630; NCT02557854).

The NCT00949325 phase I/II trial has been performed to test the ability of temsirolimus (CCI779; an mTOR inhibitor) to potentiate the cytotoxic effect of liposomal doxorubicin [11]. This trial, which enrolled 15 bone and soft-tissue sarcoma patients (including one patient with HGOS), showed that the combination of liposomal doxorubicin with temsirolimus can be safely administered to heavily pretreated patients with recurrent or refractory bone and soft tissue sarcomas and that the toxicity of this combination was manageable and reversible [11]. The NCT00019630 phase I trial has been completed, but no study results have been posted yet.

The NCT02557854 phase I trial is not open yet for recruitment. Its estimated primary completion date is December 2018.

Although there is some evidence that doxil could be a possible alternative drug to native doxorubicin, it is still too early to define its actual value for treating doxorubicin-resistant HGOS patients.

More recently, aldoxorubicin (formerly DOXO-EMCH, then renamed as INNO-206), an albuminbinding prodrug of doxorubicin with acid-sensitive properties, has emerged as an agent superior to native doxorubicin in several preclinical tumour models [12]. Phase I and II studies showed that aldoxorubicin was able to induce tumour regression in breast cancer, small cell lung cancer, and sarcomas [13]. Aldoxorubicin also appeared to be more effective than doxorubicin in advanced soft-tissue sarcoma patients [14], despite producing a higher incidence of grade 3-4 neutropenia [15]. In the past years, trials based on the use of aldoxorubicin alone or in combination with other chemotherapeutic drugs have been launched for treating patients with advanced solid tumours and 
sarcomas. However, most of them have been either completed without providing results (ClinicalTrials.gov Identifiers: NCT01337505, NCT01673438) or are active but not recruiting patients (ClinicalTrials.gov Identifiers: NCT02049905, NCT02235688). At present, there is one phase I/II trial (ClinicalTrials.gov Identifier NCT02235701) that is recruiting participants. Its completion date is scheduled for December 2018. This trial is aimed to investigate the safety and activity of aldoxorubicin plus ifosfamide/mesna in patients with metastatic, locally advanced, or unresectable soft-tissue sarcomas and it may provide information which can be of interest also for HGOS. However, similarly to doxil, the actual clinical value of aldoxorubicin in HGOS still needs to be established.

Other chemically modified doxorubicins have recently been developed and proved to be effective against drug-resistant tumour cells overexpressing $\mathrm{ABCB} 1[16,17]$. A mitochondriatargeting doxorubicin recently proved to be more effective and less cardiotoxic than doxorubicin in murine osteosarcoma preclinical models and to overcome the ABCB1-mediated doxorubicin resistance in HGOS cell lines [16].

Other compounds obtained by combining doxorubicin with H2S donors through an ester linkage at $\mathrm{C}-14$ showed to be less cardiotoxic to in vitro cultured cardiomyocytes and more active than the native drug also in ABCB1-overexpressing, doxorubicin-resistant human HGOS cell lines [17].

These findings indicate these doxorubicin derivatives as promising new chemotherapeutic drugs for a possible future clinical application in doxorubicin-unresponsive HGOS patients.

Very recently, the progress of nanomedicine has offered new hope for improving chemotherapeutic drug efficacy, as well as for overcoming chemotherapy resistance and reducing collateral toxicities. Nanomedicine techniques have also been applied to doxorubicin, in order to increase its in vivo stability and to control its intracellular drug release.

Although this approach has not specifically been studied in HGOS, findings provided in other human tumours have indicated that the nanodrug delivery system may be a promising selective modality to overcome the ABCB1-mediated doxorubicin resistance $[18,19]$, and to improve its treatment efficacy by simultaneously reducing the risk for cardiotoxicity [20].

As representative examples, we briefly mention here two doxorubicin nanoassemblies that have emerged as promising candidates for being transfered into clinical settings.

Using a so-called "squalenoylation" technology, doxorubicin can be chemically linked onto squalene, a natural lipid precursor of the cholesterol biosynthesis. The resulting squalenoyl doxorubicin proved to improve doxorubicin response of human tumour cells both in in vitro and in vivo experimental models, as well as to reduce the cardiotoxicity compared to the native doxorubicin [21]. 
Of particular interest for a possible clinical application in the near future is the very recent development of an injectable nanoparticle generator (iNPG) which can be loaded with poly(1glutamic acid)-conjugated doxorubicin (pDox) [22]. The resulting iNPG-pDox was able to overcome multiple biological barriers to cancer drug delivery and resistance. In fact, it has been shown that, after intravenous injection, iNPG-pDox accumulated at tumours and released pDox nanoparticles that were internalised by neoplastic cells. Intracellularly, pDox nanoparticles were transported to the perinuclear region and cleaved into doxorubicin, thereby avoiding excretion by drug efflux pumps like ABCB1 [22].

Finally, novel indications for modulating HGOS patients' treatment may derive from pharmacogenomic studies. Several tumour (somatic) and normal cell (germline) markers have been suggested to modulate responsiveness to conventional chemotherapeutic drugs in HGOS. Although pharmacogenomic studies in HGOS are still at their beginning, some interesting evidence has already emerged (reviewed in: [23]).

The role of somatic ABCB1 overexpression in HGOS cells for doxorubicin resistance is well understood, whereas there is less consensus regarding the impact of germline $\mathrm{ABC}$ transporter polymorphisms for patients with HGOS ([23] and references therein). Among the transporter genes involved in doxorubicin transport, ABCB1_rs1128503 was reported to correlate variably with response to combined doxorubicin chemotherapy and survival in HGOS ([23] and references therein). ABCC2_rs717620 was associated with poor histological response and a decreased risk for hematological or liver toxicity, and ABCC3_rs939338 with worse outcome ([23] and references therein). Moreover, in a recent study, ABCC2_rs2273697 was associated with poor survival and an increased risk for hematological toxicity [24]. However, it should be noted that in none of these studies toxicity was evaluated in relation to doxorubicin cycles alone. Therefore, these polymorphisms might be associated with the combined effect of all the drugs administered.

In one study, evaluating toxic events also in relation to doxorubicin alone, the variant GSTP1_rs1695 was associated with increased risk of leukopenia and cardiotoxicity [25]. Other genes with reported clinical impact in HGOS patients in relation to doxorubicin belong mainly to several DNA repair pathways and drug metabolising enzymes ([23] and references therein).

Recent pharmacogenetic investigations have tried to identify genomic risk factors for anthracycline-induced cardiotoxicity in children ([26] and references therein). Although several germline variants have been reported in transporter, carbonyl reductase genes and the hyaluron synthase 3 gene, none of them can currently be used in clinical routine to tailor doxorubicin therapy. More studies are necessary to identify those genomic variants that are invariably 
associated with toxicity caused by doxorubicin treatment in HGOS patients, in order to suggest them for either modified doxorubicin or alternative drugs.

Although almost all the described genomic variants need to be functionally characterised before their possible translation to the clinical setting, once validated, they will drive the transition to genetically guided decisions for personalised therapies. This approach, using the patients' pharmacogenomic background to optimise the treatment efficacy, is expected to overcome the present limitations of standard clinical procedures, which modulate drug dosage on the basis of patients' body surface area and age without considering the fact that patients inherently have different capabilities to metabolise and respond to chemotherapeutic agents.

In conclusion, since new promising agents and therapeutic strategies, which may overcome resistance against doxorubicin in HGOS and other musculo-skeletal tumours, are currently under development and evaluation, it is possible to speculate that outcomes of these patients will reasonably improve in the near future.

\section{References}

1. Hattinger CM, Fanelli M, Tavanti E et al. Advances in emerging drugs for osteosarcoma. Expert Opin Emerg Drugs 1-20 (2015).

2. Hattinger CM, Pasello M, Ferrari S, Picci P, Serra M. Emerging drugs for high-grade osteosarcoma. Expert Opin Emerg Dr 15(4), 615-634 (2010).

3. Fanelli M, Hattinger CM, Vella S et al. Targeting ABCB1 and ABCC1 with their Specific Inhibitor CBT-1(R) can Overcome Drug Resistance in Osteosarcoma. Curr Cancer Drug Targets 16(3), 261-274 (2016).

4. Gatti L, Beretta GL, Cossa G, Zunino F, Perego P. ABC transporters as potential targets for modulation of drug resistance. Mini Rev Med Chem 9(9), 1102-1112 (2009).

5. Brozik A, Hegedus C, Erdei Z et al. Tyrosine kinase inhibitors as modulators of ATP binding cassette multidrug transporters: substrates, chemosensitizers or inducers of acquired multidrug resistance? Expert Opin Drug Metab Toxicol 7(5), 623-642 (2011).

6. Sero V, Tavanti E, Vella $\mathrm{S}$ et al. Targeting polo-like kinase 1 by NMS-P937 in osteosarcoma cell lines inhibits tumor cell growth and partially overcomes drug resistance. Invest New Drugs 32(6), 1167-1180 (2014).

7. Tavanti E, Sero V, Vella S et al. Preclinical validation of Aurora kinases-targeting drugs in osteosarcoma. Br J Cancer 109(10), 2607-2618 (2013). 
8. Le Deley MC, Guinebretiere JM, Gentet JC et al. SFOP OS94: a randomised trial comparing preoperative high-dose methotrexate plus doxorubicin to high-dose methotrexate plus etoposide and ifosfamide in osteosarcoma patients. Eur J Cancer 43(4), 752-761 (2007).

9. Piperno-Neumann S, Le Deley MC, Redini F et al. Zoledronate in combination with chemotherapy and surgery to treat osteosarcoma (OS2006): a randomised, multicentre, open-label, phase 3 trial. Lancet Oncol 17(8), 1070-1080 (2016).

10. Skubitz KM. Phase II trial of pegylated-liposomal doxorubicin (Doxil (TM)) in sarcoma. Cancer Invest 21(2), 167-176 (2003).

11. Thornton KA, Chen AR, Trucco MM et al. A dose-finding study of temsirolimus and liposomal doxorubicin for patients with recurrent and refractory bone and soft tissue sarcoma. Int $\mathbf{J}$ Cancer 133(4), 997-1005 (2013).

12. Kratz F. DOXO-EMCH (INNO-206): the first albumin-binding prodrug of doxorubicin to enter clinical trials. Expert Opin Investig Drugs 16(6), 855-866 (2007).

13. Unger C, Haring B, Medinger $M$ et al. Phase I and pharmacokinetic study of the (6maleimidocaproyl)hydrazone derivative of doxorubicin. Clin Cancer Res 13(16), 4858-4866 (2007).

14. Chawla SP, Staddon AP, Baker LH et al. Phase II study of the mammalian target of rapamycin inhibitor ridaforolimus in patients with advanced bone and soft tissue sarcomas. J Clin Oncol 30(1), 78-84 (2012).

15. Mita MM, Natale RB, Wolin EM et al. Pharmacokinetic study of aldoxorubicin in patients with solid tumors. Invest New Drugs 33(2), 341-348 (2015).

16. Buondonno I, Gazzano E, Jean SR et al. Mitochondria-Targeted Doxorubicin: A New Therapeutic Strategy against Doxorubicin-Resistant Osteosarcoma. Mol Cancer Ther 15(11), 2640-2652 (2016).

17. Chegaev K, Rolando B, Cortese D et al. H2S-Donating Doxorubicins May Overcome Cardiotoxicity and Multidrug Resistance. J Med Chem 59(10), 4881-4889 (2016).

18. Wang YC, Wang F, Sun TM, Wang J. Redox-Responsive Nanoparticles from the Single Disulfide Bond-Bridged Block Copolymer as Drug Carriers for Overcoming Multidrug Resistance in Cancer Cells. Bioconjugate Chem 22(10), 1939-1945 (2011).

19. Zhao YY, Chen F, Pan YM et al. Nanodrug Formed by Coassembly of Dual Anticancer Drugs to Inhibit Cancer Cell Drug Resistance. Acs Appl Mater Inter 7(34), 19295-19305 (2015).

20. Jeyamohan P, Hasumura T, Nagaoka Y, Yoshida Y, Maekawa T, Kumar DS. Accelerated killing of cancer cells using a multifunctional single-walled carbon nanotube-based system for 
targeted drug delivery in combination with photothermal therapy. Int J Nanomed 8 2653-2667 (2013).

21. Maksimenko A, Dosio F, Mougin J et al. A unique squalenoylated and nonpegylated doxorubicin nanomedicine with systemic long-circulating properties and anticancer activity. $\mathrm{P}$ Natl Acad Sci USA 111(2), E217-E226 (2014).

22. $\mathrm{Xu} \mathrm{R}$, Zhang $\mathrm{G}$, Mai $\mathrm{J}$ et al. An injectable nanoparticle generator enhances delivery of cancer therapeutics. Nat Biotechnol 34(4), 414-418 (2016).

23. Serra M, Hattinger CM. The pharmacogenomics of osteosarcoma. Pharmacogenomics J (2016).

24. Hattinger CM, Biason $\mathrm{P}$, Iacoboni $\mathrm{E}$ et al. Candidate germline polymorphisms of genes belonging to the pathways of four drugs used in osteosarcoma standard chemotherapy associated with risk, survival and toxicity in non-metastatic high-grade osteosarcoma. Oncotarget (2016).

25. Windsor RE, Strauss SJ, Kallis C, Wood NE, Whelan JS. Germline Genetic Polymorphisms May Influence Chemotherapy Response and Disease Outcome in Osteosarcoma. Cancer 118(7), 1856-1867 (2012).

26. Kager L, Diakos C, Bielack S. Can pharmacogenomics help to improve therapy in patients with high-grade osteosarcoma? Expert Opin Drug Met 11(7), 1025-1028 (2015). 